\title{
A Concave Hull Methodology for Calculating the Crown Volume of Individual Trees Based on Vehicle-Borne LiDAR Data
}

\author{
Zhaojin Yan ${ }^{1,2,3}{ }^{-}$, Rufei Liu ${ }^{4, *}$, Liang Cheng ${ }^{1,2,3,5}$, Xiao Zhou ${ }^{1,3}$, Xiaoguang Ruan ${ }^{1,3}$ and \\ Yijia Xiao ${ }^{1,3}$ \\ 1 Jiangsu Provincial Key Laboratory of Geographic Information Science and Technology, Nanjing University, \\ 163 Xianlin Road, Nanjing 210023, China; DG1727032@smail.nju.edu.cn (Z.Y.); lcheng@nju.edu.cn (L.C.); \\ DG1727039@smail.nju.edu.cn (X.Z.); DG1727024@smail.nju.edu.cn (X.R.); \\ MG1827078@smail.nju.edu.cn (Y.X.) \\ 2 Collaborative Innovation Center for the South Sea Studies, Nanjing University, 163 Xianlin Road, \\ Nanjing 210023, China \\ 3 School of Geography and Ocean Science, Nanjing University, 163 Xianlin Road, Nanjing 210023, China \\ 4 College of Geomatics, Shandong University of Science and Technology, 579 Qianwan Port Road, \\ Qingdao 266590, China \\ 5 Collaborative Innovation Center of Novel Software Technology and Industrialization, 163 Xianlin Road, \\ Nanjing University, Nanjing 210023, China \\ * Correspondence: liurufei@sdust.edu.cn
}

Received: 31 January 2019; Accepted: 12 March 2019; Published: 14 March 2019

\begin{abstract}
Crown volume is an important tree factor used in forest surveys as a prerequisite for estimating biomass and carbon stocks. This study developed a method for accurately calculating the crown volume of individual trees from vehicle-borne laser scanning (VLS) data using a concave hull by slices method. CloudCompare, an open-source three-dimensional (3D) point cloud and mesh processing software package, was used with VLS data to segment individual trees from which single tree crowns were extracted by identifying the first branch point of the tree. The slice thickness and number to be fitted to the canopy point cloud were adaptively determined based on the change rate in area with height, with the area of each slice calculated using the concave hull algorithm with portions of the crown regarded as truncated cones. The overall volume was then calculated as the sum of all sub-volumes. The proposed method was experimentally validated on 30 urban trees by comparing the crown volumes calculated using the proposed method with those calculated using five existing methods (manual measurement, 3D convex hull, 3D alpha shape, convex hull by slices, and voxel-based). The proposed method produced the smallest average crown volume. Gaps and holes in the point cloud were regarded as part of the crown by the manual measurement, 3D convex hull, and convex hull by slices method, resulting in the calculated volume being higher than the true value; the proposed method reduced this effect. These results indicate that the concave hull by slices method can more effectively calculate the crown volume of a single tree from VLS data.
\end{abstract}

Keywords: vehicle-borne laser scanning; LiDAR data; crown volume; concave hull; adaptive slicing

\section{Introduction}

Forests are among the most valuable resources on Earth [1,2]; they comprise the earth's ecosystem together with the atmosphere, soil, and water, playing an important role as a treasure trove of terrestrial biodiversity [3-5]. The investigation of tree parameters is valuable in understanding forest dynamics [5] and is an essential component of forestry management as a key tool in sustainable 
forest development and the accurate evaluation of forest resources [6]. Tree parameters include tree height, diameter at breast height (DBH), crown diameter, and crown volume [7]. Because of the fractal nature of plants, the definition of crown volume is quite subjective [8]. Traditionally, this term has been defined as the apparent geometric volume that includes all the branches and leaves [9]. Crown volume in this study continues this definition. Crown volume is central to the estimation of biomass and carbon stocks [3,7,10-12], but has proven to be difficult for forest workers to assess because it is impossible to directly measure using field-based surveys $[10,13,14]$. However, extracting tree parameters from remote sensing data has been shown to be an effective means of acquiring such features. Jing et al. [15] proposed a method that applies multi-scale filtering and segmentation to extract high-quality individual tree crown maps from multispectral airborne imagery. Yin et al. [16] applied an object-based method for delineating old-growth larch tree crowns using Geoeye-1 imagery and quantitatively validated the correlation between delineated tree crown and base areas. Their method provided large-scale larch tree information that was applied in the renovation of historical buildings in China. Dalponte et al. [5] obtained the three-dimensional (3D) structures of tree crowns from remote sensing data gathered using airborne laser scanner (ALS) data to detect individual tree crowns and used the resulting hyperspectral images to identify tree species. Although their work provided a theoretical approach to tree crown volume calculation, they reported no quantitative calculation results.

Various types of remote sensing data, including medium- and high-resolution optical and synthetic aperture radar (SAR) images along with light detection and ranging (LiDAR) results have been used for the extraction of tree parameters [1,4,15,17-23]. Among these, LiDAR data is widely used for tree crown detection and calculation because it can produce high-density 3D data. Kato et al. [24] developed a wrapped surface reconstruction method to obtain accurate results for various conifer and deciduous species parameters (tree height, crown diameter, and crown volume) from ALS data. Korhonen et al. [10] used 3D alpha shape and 3D convex hull techniques to extract tree crown volumes from ALS data, with the results showing that the LiDAR-based estimates were highly correlated with the field-measured tree crown volumes (best $R^{2}=0.83$ ). Lecigne et al. [25] developed a voxel-based $R$ package to analyze complex tree crown arrangements from terrestrial laser scanner (TLS) data. They noted that the voxel division size had a significant influence on the calculated crown volume and that the choice of voxel size should depend on the research objectives and the quality of the TLS data.

Despite these advantages, there are some drawbacks to using ALS and TLS data to calculate tree crown volume. ALS-produced estimates are system-driven and return limited information from within and below the crown $[10,14]$ because the low sampling density of ALS data makes it impossible to express the structure in detail [14]. The TLS approach, which employs a laser scanner mounted on a ground support $[26,27]$ to acquire high-density point clouds, can be applied to the delicate modeling of buildings and trees. However, limitations in scanner range can make it necessary to repeatedly move the system over a large observation area, producing a high amount of data-matching work and reducing the processing efficiency.

Vehicle-borne laser scanning (VLS) systems, which use vehicles as platforms [28-30], can be used to produce 3D point clouds from the surrounding objects, providing an effective method for the rapid and accurate collection of tree information [31,32]. VLS is more flexible and with lower costs when compared to ALS, and VLS is faster and capable of acquiring more 3D data than TLS [33]. The relative point precision of the VLS is generally no more than $1 \mathrm{~cm}$; however, its absolute accuracy mainly depends on the GNSS-IMU navigation solution, which can reach centimeter level [33,34]. Although there is abundant literature on detecting individual trees from VLS data [12,28,31,32,35], there have been relatively few studies on the calculation of tree crown volume. Wu et al. [35] applied a voxel-based marked neighborhood searching (VMNS) method to identify trees and derive their morphological parameters (tree height, crown diameter, DBH, and crown base height). However, they omitted crown volume calculations and only reported that tree crown volume can be characterized by summing the volumes of individual voxels within the crown. 
This study developed a new approach to calculating tree crown volume from VLS data producing a concave hull method for more accurately calculating crown slice area, and an adaptive slicing method based on the rate of change in area with height. Using these tools, the slice thickness and the number of tree crown slice layers can be adaptively determined according to the shape and the size of the crown. After estimating tree crown volume using this approach, the results were compared with those achieved using conventional methods.

\section{Literature Review}

Commonly used methods for calculating tree crown volume include conventional field-based surveys, geometric calculation, and voxel-based methods.

The conventional field-based survey approach (Figure 1a) can essentially be divided into two steps. First, the crown diameters and the heights of trees are measured in the field $[9,11,26,36]$. The crown diameter is usually taken as the average of the crown diameters in the east-west and north-south directions [7,35], while the crown height is taken as the difference in height between the first branch point and the vertex of the crown $[9,35]$. Second, an appropriate geometry must be used to fit the crown shape, with the volume of the geometry used to calculate the crown volume [9,26]. Ellipsoids are most widely used to fit crown shapes $[10,26,36]$.
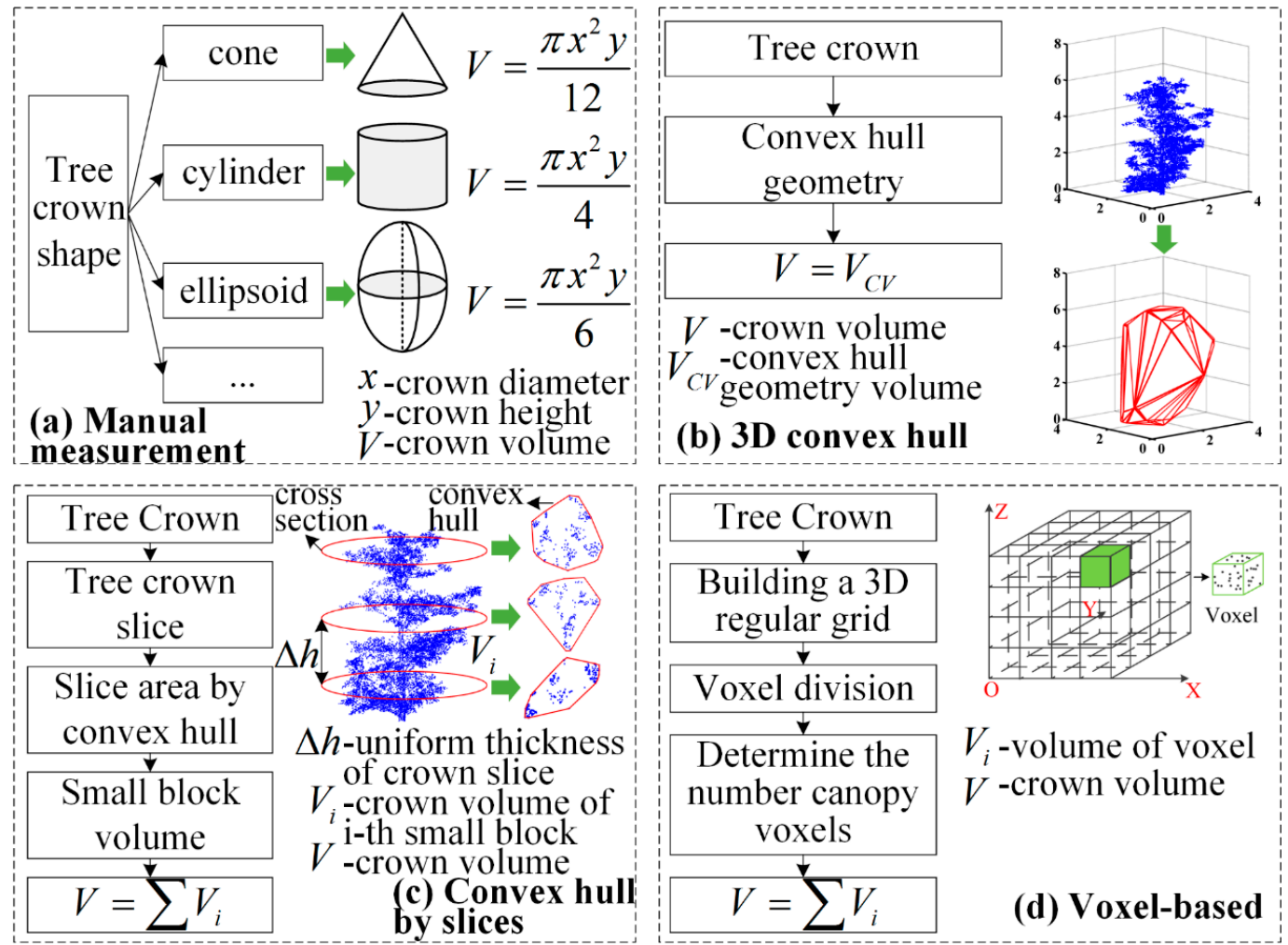

Figure 1. Schematics of four tree crown volume calculation methods: (a) manual measurement, (b) three-dimensional (3D) convex hull, (c) convex hull by slices, and (d) voxel-based.

The geometric calculation method involves construction of a geometry based on 3D scatter data obtained by applying a LiDAR system to the crown; the volume of the geometry is used to calculate the tree crown volume. Gupta et al. [37] reconstructed the crown structure of individual trees using the 3D convex hull algorithm (Figure 1b), an approach that has been applied in a number of studies [9-11,38]. Fernández-Sarría et al. [9] improved the overall 3D convex hull algorithm by segmenting using height intervals of $5 \mathrm{~cm}$, calculating the volumes of small blocks by applying 3D convex hulls, then summing the small block volumes to obtain the crown volume. Similarly, two-dimensional (2D) convex hulls 
(Figure 1c) have been used to calculate the planar areas of individual crown slices, which are then divided into small blocks that are summed to produce the tree crown volume [7,31].

Another commonly used geometric calculation method is the alpha shape approach [10,26,39-44], in which geometric reconstruction is performed from a discrete set of spatial points (2D or 3D), i.e., contours are extracted from a set of unordered points. The alpha shape of a finite point set $S$ is a closed polyhedron that is uniquely determined by the constituents of $S$ and $\alpha$, which is a parameter determined by the level of detail of the polyhedron (Figure 2). The principle behind the construction of the polyhedron can be imagined as a circle with a radius $\alpha$ rolling along the exterior of $S$ [39] (Figure 2a). For a sufficiently large value of $\alpha$, the path of the circle will not go inside $S$ and the trace of its scroll will therefore map the boundary line of $S$. The alpha shape approach can be regarded as a generalization of the convex hull method: as $\alpha \rightarrow \infty$, the alpha shape converges to a convex hull [39,43] (Figure 2c). By gradually decreasing the value of $\alpha$, a more vivid boundary of $S$ can be generated (Figure 2a,b). Vauhkonen et al. $[39,40,42,44]$ applied the alpha shape approach to perform a number of experiments on the segmentation and description of individual trees and found that the alpha shape deteriorated when the LiDAR point density was low [44]. Korhonen et al. [10] used the 3D alpha shape technique to calculate crown volumes from LiDAR point cloud data and concluded that this approach produces more accurate crown volumes than ellipsoid-fitting using the conventional method.

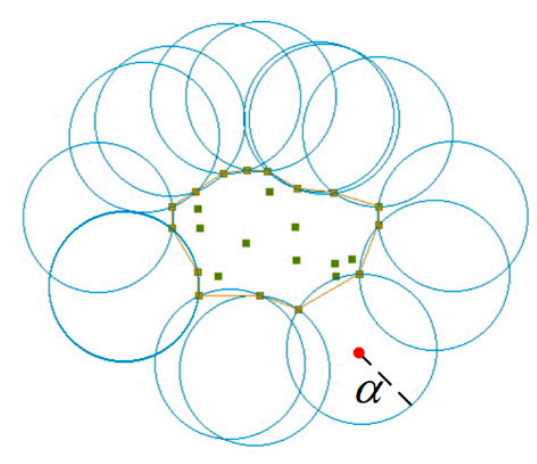

(a) $R=\alpha$

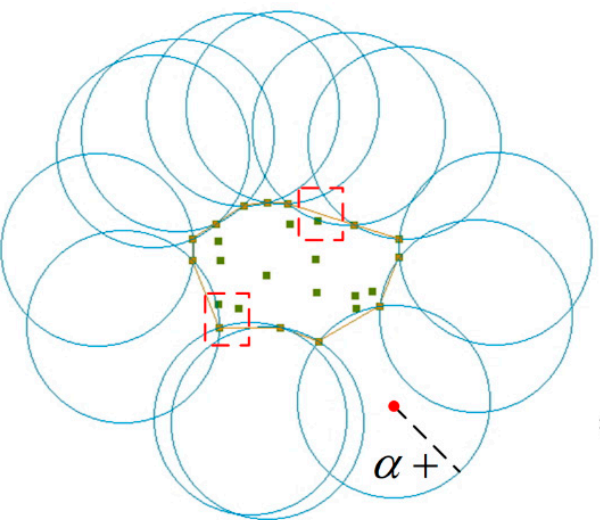

(b) $R=\alpha+$

\section{Convex hull}

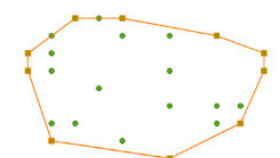

$* \alpha<\alpha+<\infty$

(c) $R=\infty$

Figure 2. Schematic of the 3D alpha shape method. For a given set of discrete points, the alpha shape extracted using the alpha value $\alpha$ (a) and the alpha shape extracted using the alpha value $\alpha+(\mathbf{b})$. As the alpha value increases $(\alpha<\alpha+)$, the fineness of the extracted alpha shape decreases (red dotted box in $(\mathbf{b})) . \alpha \rightarrow \infty(\mathbf{c})$, in which the extracted alpha shape is a convex hull.

The voxel-based method (Figure 1d) uses a regular 3D grid to organize a discrete crown point cloud. Depending on the range of this point cloud, extreme values in the $\mathrm{X}-, \mathrm{Y}-$, and $\mathrm{Z}$-directions are determined and the grid is divided according to the cell spacing on each coordinate axis to produce a set small cubes called voxels [32,35]. The volumes of the voxels containing at least one data point are summed to obtain the crown volume $[9,25,45]$. Hosoi and Omasa [46] applied a voxel-based method to reconstruct a precise 3D model of a crown that included its interior. Wu et al. [35] used the voxel-based method to separate the crown and trunk of a single tree from VLS data and estimated its morphological parameters (tree height, crown diameter, crown height, and DBH); they observed that the method can be used to calculate crown volume.

The above methods have several drawbacks. Conventional field-based surveys, for example, require a high amount of manpower and material resources, which is expensive and time-consuming [36,47]. Crown volumes calculated using 3D convex hulls do not include gaps within the crown and therefore generally overestimate its volume [10,24]. Although slice-based volume calculation reduces the estimation error of global 3D convex hull approaches [9], there is no uniform method for producing segmentation $[11,26]$. Furthermore, methods for calculating crown 
slice area based on 2D convex hulls have shown to be not very effective [48]. Theoretically, the alpha shape approach uses an optimal alpha value to approximate crown shape; however, a solution to this optimal value is very difficult $[10,44]$. Although the voxel-based method is considered to produce the best approximations of true crown shape $[9,25,45,46]$, the calculated crown volume depends on the voxel size and the approach involves a large cost in terms of time [9,49].

Given the problems described above, we propose a novel method for accurately calculating crown volume, in which a concave hull algorithm is used to calculate the crown slice area and an adaptive slicing method is then used to perform point cloud slicing. The results produced by the proposed method were compared with those obtained using five existing approaches (manual measurement, 3D convex hull, 3D alpha shape, convex hull by slices, and voxel-based).

\section{Materials and Methods}

\subsection{Field Data Collection}

Thirty trees in the city of Qingdao, Shandong Province, China were selected for this study: 20 Platanus orientalis and 10 Ginkgo biloba, both of which are common, tall, deciduous street tree species [50,51]. P. orientalis grows fast and produces large, approximately oval canopies [52]. G. biloba is one of the oldest living tree species [51] and has a conical crown shape [52]. Tree point cloud data were collected using the Vsurs-Q VLS system, which was jointly developed by the Shandong University of Science and Technology and Qingdao Xiushan Mobile Measurement Co., Ltd. The Vsurs-Q VLS system uses a motorcycle as a platform and employs laser scanners, an integrated navigation system, panoramic cameras, and various sensors (Figure 3). It has been applied to topographic mapping, ancient building protection, streetscape image acquisition, and urban component mapping. Because the Vsurs-Q system is compact, it offers high maneuverability, convenient data collection, and wide applicability. Vsurs-Q uses a Faro X130 laser scanner (technical parameters given in Table 1).

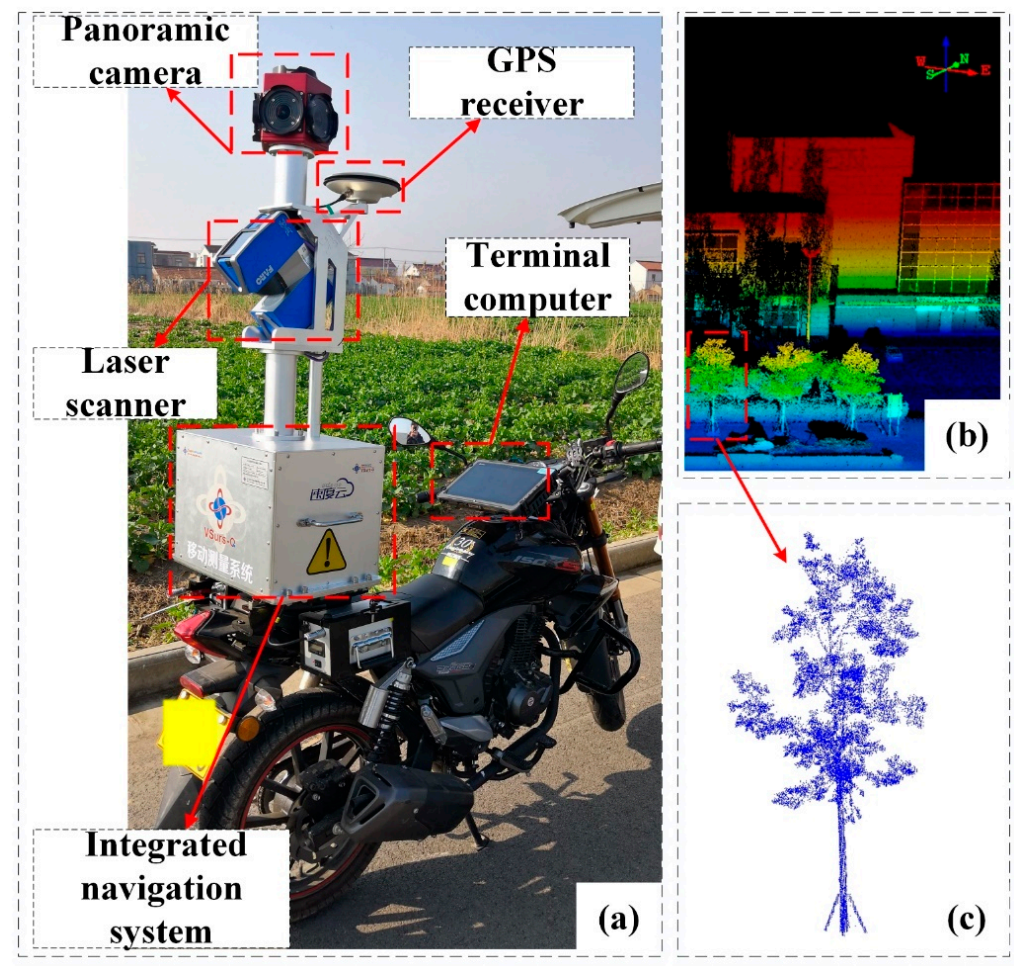

Figure 3. Vsurs-Q vehicle-borne laser scanning (VLS) system: (a) system sensors, including a panoramic camera, laser scanner, GPS receiver, integrated navigation system, and terminal computer; (b) VLS data collected using the Vsurs-Q VLS system; (c) VLS data for a single tree. 
Table 1. Main technical parameters of the Faro X130 laser scanner.

\begin{tabular}{cc}
\hline Indicator & Value \\
\hline Scanning distance & $\leq 130 \mathrm{~m}$ \\
Angle of field of view & Vertical angle $305^{\circ}$ \\
Horizontal angle $360^{\circ}$ \\
Accuracy of measurement & $\pm 2 \mathrm{~mm}$ accuracy of measurement at a scanning distance of $25 \mathrm{~m}$ \\
Angle resolution & Vertical resolution $0.009^{\circ}$ \\
Line frequency & Horizontal resolution $0.009^{\circ}$ \\
Scanning rate & $97 \mathrm{~Hz}$ \\
\hline
\end{tabular}

\subsection{Calculating Crown Volume Using VLS Data}

Although a number of methods for the segmentation of individual trees using VLS data have been reported in the literature $[12,31,32,35,49]$, this study focused on methods for calculating crown volume, and therefore, did not apply an individual tree segmentation algorithm. Instead, the work of segmentation of individual trees was performed manually using CloudCompare, an open-source 3D point cloud processing software package (Figure 4a). In addition to its general point cloud processing capabilities (browsing, editing, and thinning), CloudCompare includes many advanced algorithms, such as registration, resampling, statistics computation, and interactive or automatic segmentation $[53,54]$. The principal method flow undertaken in this study (Figure 4) involved four main parts: (1) segmentation of individual trees using the VLS data; (2) tree crown extraction; (3) calculation of crown volume using the proposed concave hull by slices algorithm; and (4) comparison of the results with those produced by the five existing methods.

\subsubsection{Tree Crown Extraction}

The first branch point of a tree is the division point between the crown and the trunk [11,43,55]. In other words, a tree point at a height greater than that of the first branch point belongs to the crown; otherwise, it is a trunk point. The first branch point is determined by assuming that the horizontal projection characteristics of the tree will change at this point, with the horizontal projection area above the first branch point larger than the area below it. By identifying the first branch point of a tree, its crown can be extracted (Figure $4 \mathrm{~b}$ ), thereby providing data support for the calculation of the crown volume. Using this principle, its height can be manually determined using CloudCompare software and the point cloud output above it can be defined as the tree crown point cloud.

The flowchart of the first branch point identification algorithm is shown in Figure 5. First, the tree point cloud was imported into the CloudCompare software, and the point cloud within a certain height range containing the first branch point was manually selected as the candidate point cloud. The height range of the candidate point cloud was determined manually, as shown in the red solid line box in Figure 5. Then, the candidate point cloud was subdivided into 3D cells. The cell size was determined empirically and can also be manually tuned repeatedly. The horizontal projected area of each layer was calculated, which is the number of cells containing the crown points. The area of the adjacent layer was compared from bottom to top to determine the layer in which the first branch point is located. When the area of the upper layer was larger than that of the lower layer, and the difference in area between the upper and lower layers was greater than a certain threshold, it was considered that the first branch point was located in the lower layer, and the height of the lower layer was the height of the first branch point. 


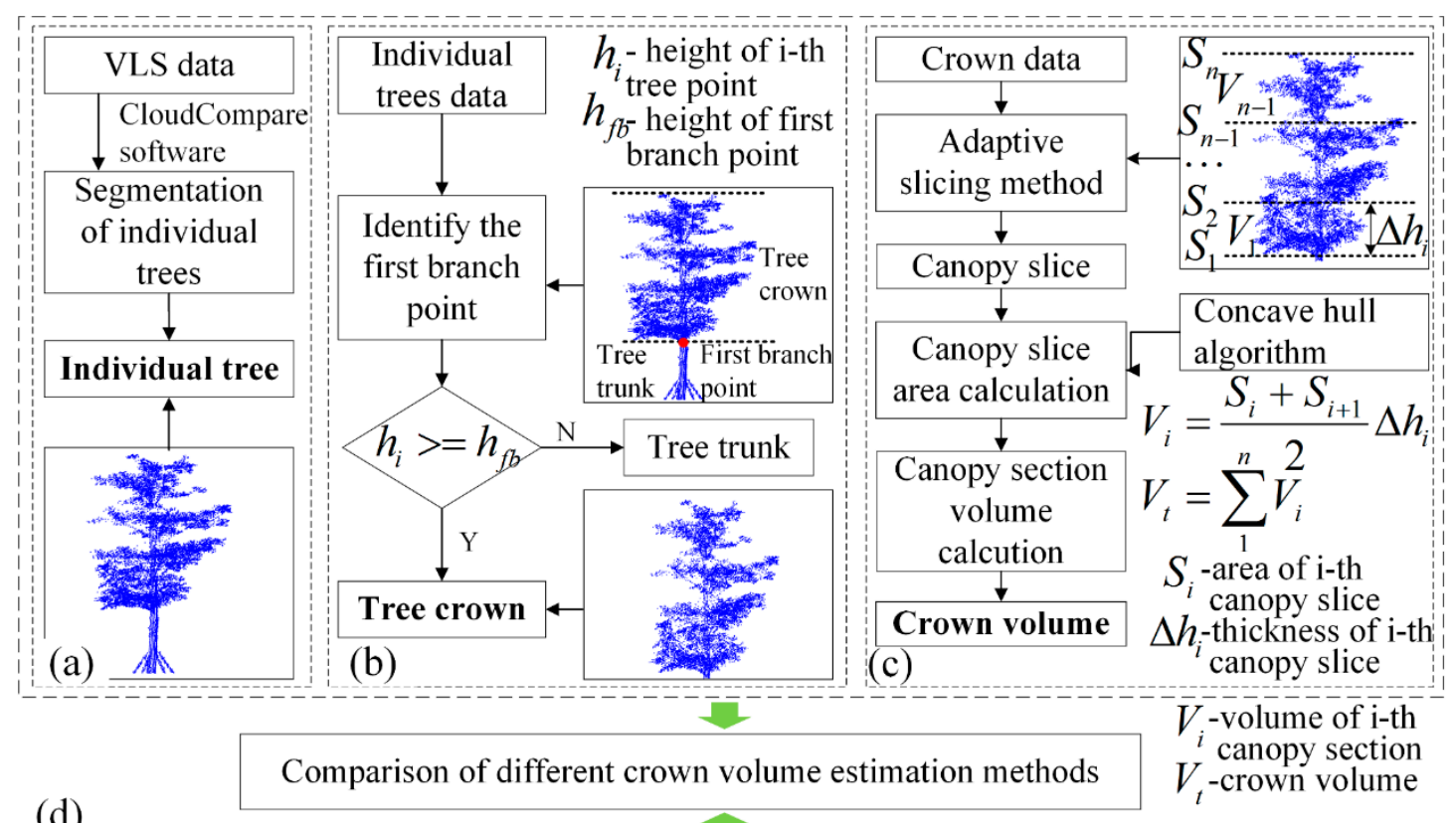

(d)

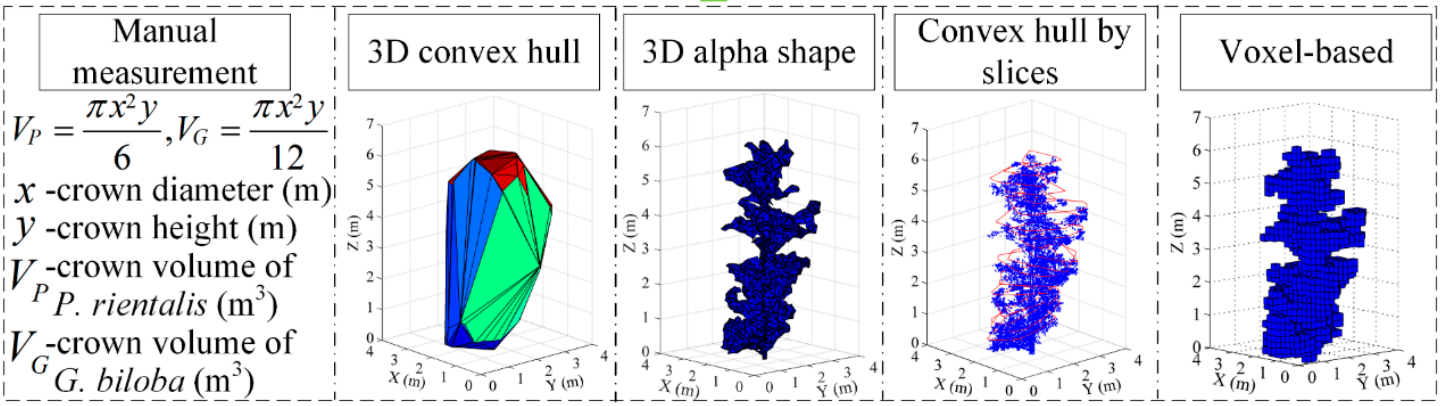

Figure 4. Flow chart of study methodology. CloudCompare software was used with VLS data to segment individual trees (a) from which single tree crowns were extracted by identifying the first branch point of the tree (b). Crown volumes were calculated based on concave hull by slices method (c), and the results were compared with those of the existing five methods (d).

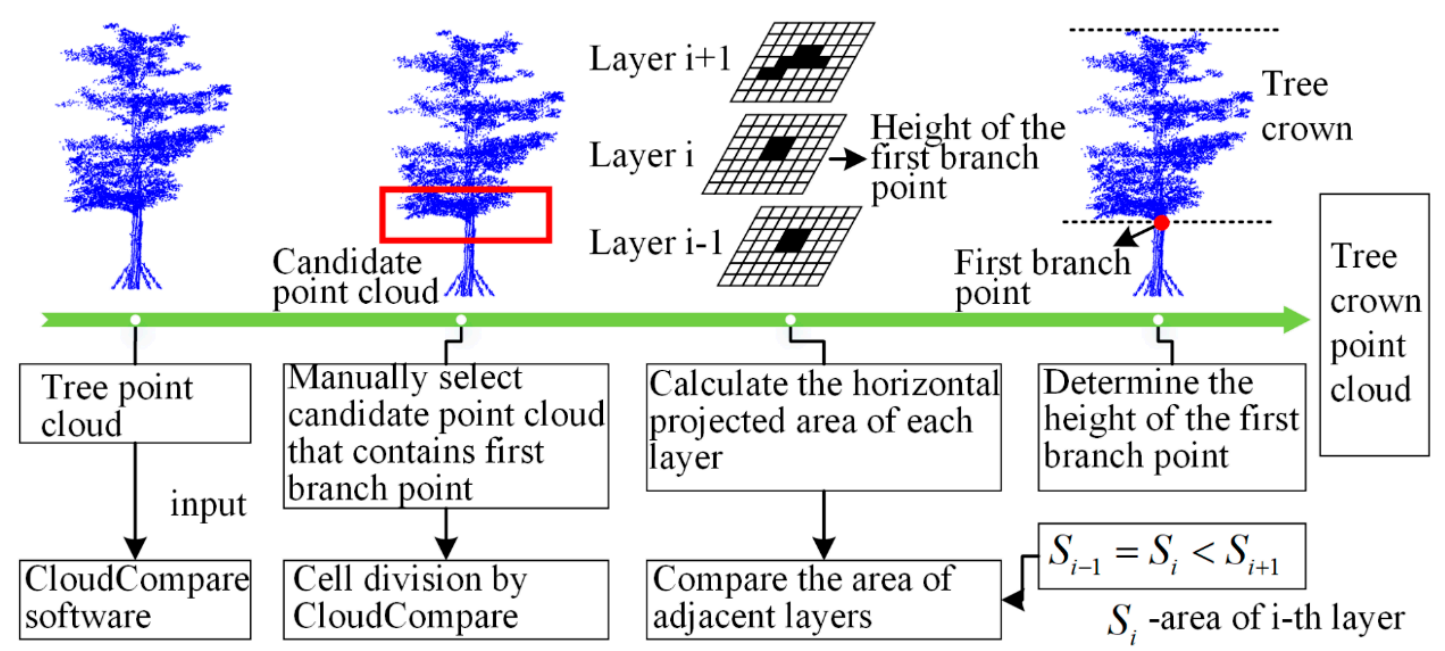

Figure 5. Flow chart of the first branch point identification algorithm. 


\subsubsection{Slice Area Calculation Using Concave Hull Algorithm}

For discrete points on a given plane, the convex hull approach is a commonly used method to calculate the plane area; however, the boundary of the convex hull polygon does not really represent the contour of the discrete points, which is especially evident in the tree point cloud [48]. Due to existence of holes and gaps in the crown point cloud, if the convex hull is used to calculate the horizontal area of the crown slice, its area will be overestimated. The concave hull algorithm has been proposed to better describe the area formed by discrete points on a given plane [48,56,57]. A set of discrete points on a given plane has a unique convex hull, but generally do not have a unique concave hull. Therefore, a method for determining candidate concave hull has been proposed, which is the $\mathrm{k}$-nearest neighbor concave hull algorithm [48]. As the number of neighbor points ( $\mathrm{k}$ value) increases, if the vertices of the candidate concave hull are sequentially connected to form a closed polygon, the concave hull that satisfies the requirements is obtained. The following steps are used by the concave hull approach to sum the areas of crown point cloud slices:

Step 1: The point $\mathrm{A}\left(x_{a}, y_{a}\right)$ with the smallest $y$ value in a plane point set is used as the initial detection point, with Point $\mathrm{O}$, whose coordinates satisfy the following conditions, used as an auxiliary point:

$$
x_{o}=x_{a}-1, y_{o}=y_{a}
$$

Step 2: An initial $\mathrm{k}$ value of not less than three is determined and then the $\mathrm{k}$ points $\left(P_{1}, P_{2}, \cdots, P_{k}\right)$ nearest to point $A$ are selected. This is illustrated in Figure 6a, with points B, C, and D selected for $\mathrm{k}=$ 3. For each $\mathrm{k}$, the three points $\mathrm{O}, \mathrm{A}$, and $P_{k}$ are connected in the clockwise direction to form the angle $O A P_{k}$ and all k points are traversed to find the point with the maximum value of $O A P_{k}$, which is set as the second point of the concave hull. In the Figure $6 a$, this is point D.

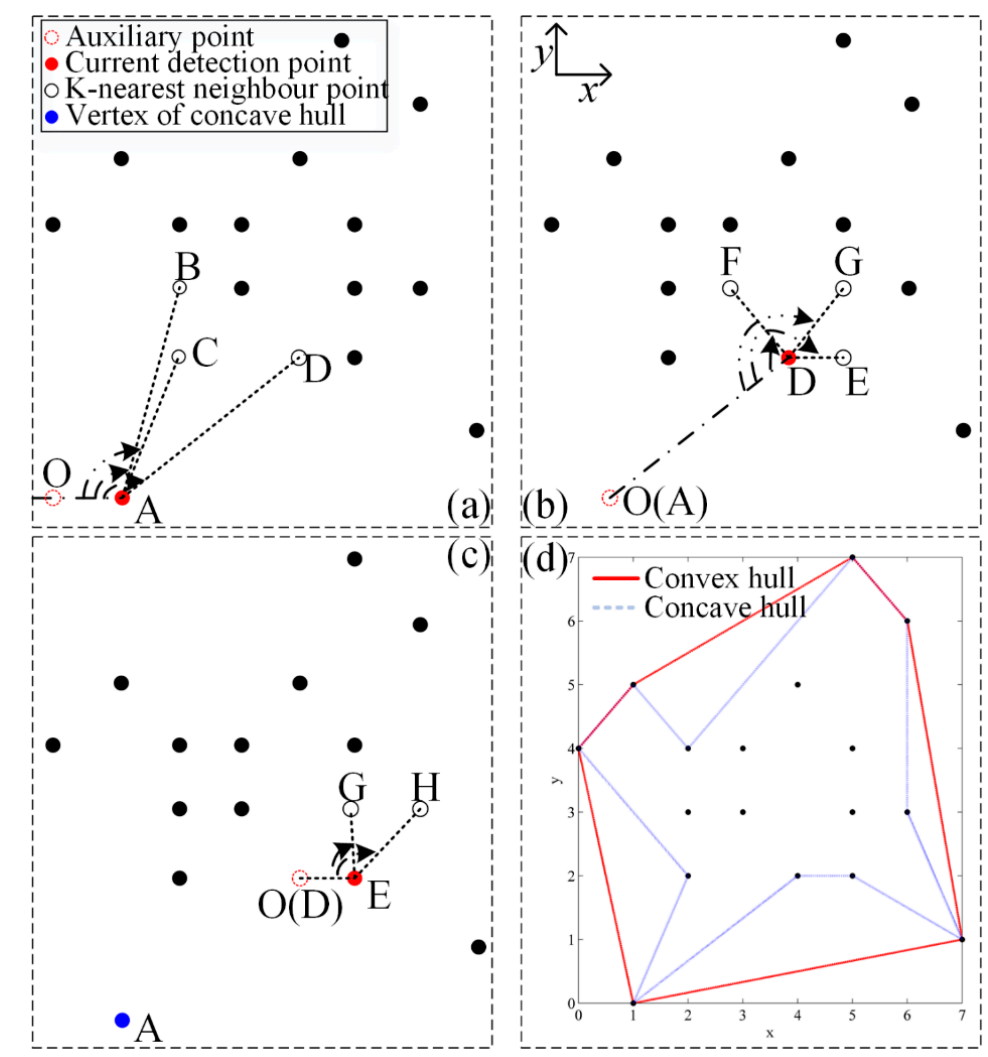

Figure 6. The principle of the k-nearest neighbor concave hull algorithm as illustrated in [48] for $(\mathbf{a}-\mathbf{c}) \mathrm{k}=3$ (locating the three closest points to the selected point); (d) provides an example contrasting a convex and concave hull for a given set of points. 
Step 3: Replacing the vertex point $\mathrm{A}$ by $\mathrm{O}$ and using the current vertex (point $\mathrm{D}$ ) as the detection point, Step 2 is repeated. In Figure $6 \mathrm{~b}$, point $\mathrm{E}$ is selected in this process. The next vertex (Figure $6 \mathrm{c}$, point $\mathrm{H}$ ) is selected in a similar manner. In this case, however, point $\mathrm{H}$ cannot be directly regarded as a concave hull vertex and it is necessary to check whether the line segment $\mathrm{EH}$ intersects with the previously formed concave hull line segments that are not adjacent to $\mathrm{EH}$. If not, $\mathrm{H}$ is designated as the vertex of the concave hull; otherwise, it is removed from the k-nearest neighbor point set and the remaining point that generates the maximum angle is selected if it satisfies the edge condition. These steps are repeated until the subset of k-nearest neighbor points of $E$ satisfying the edge condition is identified. If this subset is empty, then the value of $k$ is iterated $(k=k+1)$ and all of concave hull points are recalculated.

Step 4: If a concave hull is formed using the process in Steps 1-3 with a vertex is the initial point A, a closed concave hull has been formed and the loop terminates, although the final solution has not necessarily been reached because it is also necessary to judge whether the concave hull encloses all the data points within a given plane of the hull. The interiority of each point is determined by counting the number of intersections of a ray generated from the point with the surface of the concave hull; if it is odd, the point is inside the concave hull; if it is even, it is outside. If there is at least one point outside of the concave hull, the concave hull construction is considered to have failed and it is necessary to increase the $\mathrm{k}$ value and restart the calculation.

Step 5: Once the concave hull has been built, its vertices are arranged counterclockwise. For the vertex coordinates $\left(x_{1}, y_{1}\right),\left(x_{2}, y_{2}\right), \cdots,\left(x_{i}, y_{i}\right), \cdots,\left(x_{n}, y_{n}\right)$, the formula for calculating the concave hull area is:

$$
S=\frac{1}{2} \sum_{i=1}^{n-1}\left(y_{i}+y_{i+1}\right)\left(x_{i}-x_{i+1}\right),
$$

where $S$ is the area of the crown point cloud slice calculated based on the concave hull algorithm, $\left(x_{i}, y_{i}\right)$ are the coordinates of the i-th vertex of the concave hull formed by the crown point cloud slice, and $n$ is the number of concave hull vertices.

\subsubsection{Adaptive Slicing of Tree Crown}

Differences in species, growing environment, planting time, and other factors lead to significant variations in crown shape and size among individual trees of the same or different species, making it unsuitable to slice the crown point cloud in the vertical direction with a uniform thickness [58]. To overcome this, we propose a slicing method that adaptively determines the number of layers and the thickness of each layer based on the shape and size of the crown point cloud. Slices are only created when the slice area in the vertical direction changes, ensuring that the horizontal projection area of the point cloud in the same slice is similar and that the horizontal projection area of the point cloud in different slices is significantly different. By reducing the number of slice layers, the proposed method ensures that the thickness of each slice and the number of slice layers are well-adapted to the shape and size of the tree crown itself, thereby improving the slicing rationality of the point cloud in the vertical direction. The adaptive slicing method algorithm employs the following steps (Figure 7):

Step 1: Uniform-thickness tree crown slices are generated by dividing the point cloud into $m$ layers in the vertical direction, each with an initial layer thickness of $t_{i}=t_{0}(i=1,2, \cdots, m)$. A value of $t_{0}=5 \mathrm{~cm}$ was used in this study. Because construction of the concave hull requires at least three points, a slice containing less than three points is merged with its upper adjacent slices until the resulting slice contains at least three points. After recording the thickness and point cloud data, the slice area of each point cloud layer is calculated using the concave hull method and recorded as $S_{i}$.

Step 2: The vertical rate of change in the slice area is calculated and adjacent slices with minimal difference in area are combined into a single slice to fit the crown. First, the areas of the slices are 
classified with respect to the standard deviation in area. Taking the average of all slice areas as the starting point and the standard deviation as the spacing, the i-th slice can be categorized as follows:

$$
C_{i}=\left\{\begin{array}{l}
\operatorname{INT}\left(\frac{S_{i}-S_{\text {mean }}}{S t d}\right)+1, \text { if } S_{i}-S_{\text {mean }} \geq 0 \\
\operatorname{INT}\left(\frac{S_{i}-S_{\text {mean }}}{S t d}\right)-1, \text { if } S_{i}-S_{\text {mean }}<0
\end{array}\right. \text { ， }
$$

where $C_{i}$ is the category number in which the $\mathrm{i}$-th slice is located, $S_{i}$ is its slice area, $S_{\text {mean }}$ is the arithmetic mean of all tree crown slice areas (calculated as $S_{\text {mean }}=\frac{\sum_{i=1}^{N} S_{i}}{N}$, where $N$ is the layer number), $S t d$ is the standard deviation of all tree crown slice areas (calculated as $S t d=\sqrt{\left.\frac{1}{N-1} \sum_{i=1}^{N}\left(S_{i}-S_{\text {mean }}\right)^{2}\right)}$, and $\operatorname{INT}()$ is a truncation function that leaves only the integer part of the argument. If adjacent slices $\mathrm{i}$ and $\mathrm{i}+1$ belong to the same category, they are merged:

$$
C_{i+1}=C_{i}
$$

where $C_{i}$ and $C_{i+1}$ are the category numbers of slices $i$ and $i+1$, respectively. If the slice areas of layers $i$ and $i+1$, satisfy Equation (4), the point cloud is considered consistent between the two layers and they are merged into a single layer, $i$, of thickness $T_{\mathcal{C}}=T_{i}+T_{i+1}$, where $T_{i}$ and $T_{i+1}$ are the thicknesses of layers $i$ and $i+1$, respectively. Otherwise, the two layers are left unmerged and the slice areas of layers $i+1$ and $i+2$ are compared.

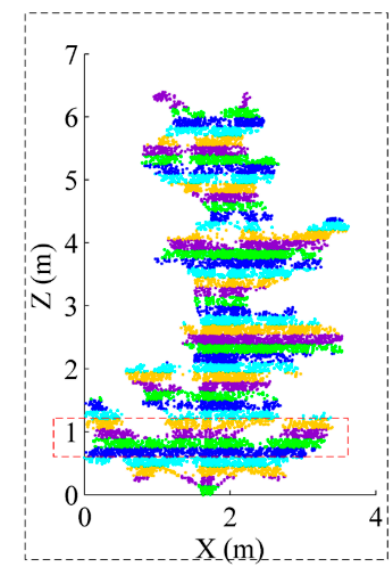

Sliced point cloud of tree crown with uniform thickness $t$ $(t=0.15 \mathrm{~m}, N=42)$

$\mathrm{N}$-the number of layers

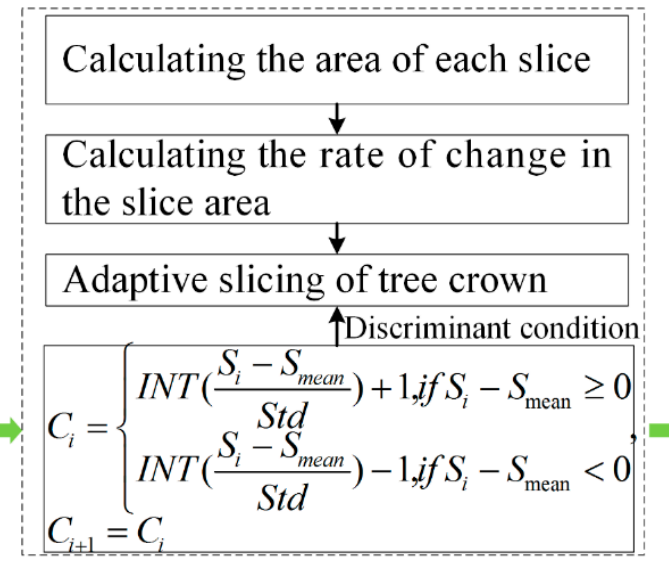

$S_{i}$ is the area of the i-th canopy slice $S_{\text {mean }}$ is the arithmetic mean value of all tree crown slice areas

$S t d$ is the standard deviation of all tree crown slice areas $C_{i}$ is the category number of $\mathrm{i}$-th tree crown slice area

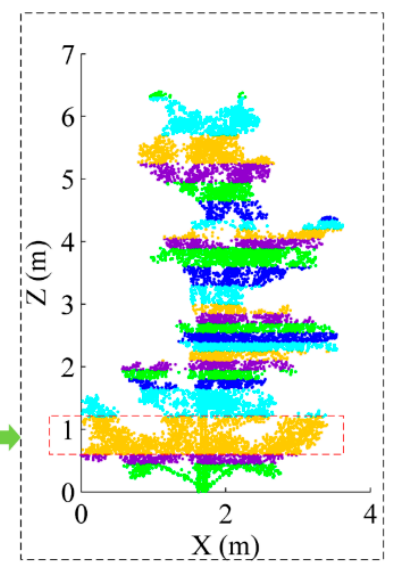

The adaptive slicing result $(N=24)$

Figure 7. Adaptive slicing of a tree crown.

Step 3: The tree crown is sliced using an adaptive thickness approach. First, the slices meeting the combined conditions in Step 2 are combined from bottom to top and sequentially numbered from bottom to top, with the layer number, slice area, and slice thickness of each crown slice recorded as:

$$
l_{i}=\left\{\left(i, S_{i}, t_{i}\right), i=1,2, \cdots, n\right\},
$$

where $l_{i}$ is the $\mathrm{i}$-th slice, $i$ is the layer number, $S_{i}$ is the slice area of the $\mathrm{i}$-th slice, $t_{i}$ is the slice thickness of the $\mathrm{i}$-th slice, and $n$ is the total number of slices. 


\subsubsection{Tree Crown Volume Calculation}

Using the proposed adaptive slicing method described in the previous sub-section, the tree crown point cloud is divided into $N$ layers that sum to the total crown volume. The volume of each layer is calculated as follows:

$$
V_{i}=\frac{S_{i}+S_{i+1}}{2} \Delta h_{i}
$$

where $V_{i}$ is the volume of the $\mathrm{i}$-th layer, each $S$ is the area of the concave hull formed by a given plane of points, where $S_{i}$ and $S_{i+1}$ are the areas of the lower and uppers borders of the i-th sub-volume, and $\Delta h_{i}$ is the slice thickness of the $i$-th layer.

The volume of the entire crown, $V_{t}$, is then simply the sum of the layer volumes:

$$
V_{t}=\sum_{i=1}^{n} V_{i}
$$

where $V_{i}$ is the volume of the $\mathrm{i}$-th layer and $n$ is the number of horizontal sections.

\subsection{Comparison of Five Crown Volume Calculation Methods}

To evaluate the accuracy of the proposed method in calculating crown volumes from VLS data, its performance was compared with those of the five existing methods. Linear regression was used to measure the strength of relationship (determination coefficient, $R^{2}$ ) between crown volumes calculated by the existing method and the proposed method. We also applied $t$-tests to compare the calculation results of different methods. All methods and analysis were conducted using MATLAB 2018 (The MathWorks Inc., Natick, MA, USA).

Manual measurement: the crown volume, $V_{P}$, of the approximately oval P. orientalis $[11,52]$ was calculated using:

$$
V_{P}=\frac{\pi x^{2} y}{6}
$$

where $x$ is the crown diameter and $y$ is the crown height.

The crown volume, $V_{G}$, of the approximately conic $G$. biloba [52] was calculated using:

$$
V_{G}=\frac{\pi x^{2} y}{12}
$$

where $x$ and $y$ are the same as in Equation (8).

3D convex hull: the entire crown was represented as a 3D convex hull geometry.

3D alpha shape: the entire crown was represented using a 3D alpha shape geometry.

Convex hull by slices: the crown was divided into slices along the vertical axis from the bottom to the top of the tree in $20 \mathrm{~cm}$ intervals. The area of each slice was defined as the area bounded by the 2D convex hull of points on the plane corresponding to the slice and the volume of the slice was calculated as the volume of a truncated cone stretching from the top to the bottom of the slice [11]. The volume of the overall crown was then calculated as the sum of the slice volumes.

Voxel-based method: The crown points were divided into voxels of size $0.2 \mathrm{~m} \times 0.2 \mathrm{~m} \times 0.2 \mathrm{~m}$ and the crown volume was calculated as product of the voxel size and the number of voxels containing at least one tree count LiDAR point $[25,45,59]$.

\section{Results and Discussion}

The crown volumes of the 30 sample trees calculated using the six methods differed significantly by tree species and stage of growth (Table 2), with a manual measurement volume range (standard deviation) of 3.84-85.54 $\mathrm{m}^{3}\left(16.21 \mathrm{~m}^{3}\right), 3 \mathrm{D}$ convex hull range of $4.30-66.58 \mathrm{~m}^{3}\left(14.54 \mathrm{~m}^{3}\right), 3 \mathrm{D}$ alpha shape range of $0.81-50.28 \mathrm{~m}^{3}\left(9.48 \mathrm{~m}^{3}\right)$, convex hull by slices range of $2.17-38.90 \mathrm{~m}^{3}\left(8.74 \mathrm{~m}^{3}\right)$, voxel-based 
range of $1.56-14.96 \mathrm{~m}^{3}\left(4.14 \mathrm{~m}^{3}\right)$, and concave hull by slices range of $1.03-23.99 \mathrm{~m}^{3}\left(5.09 \mathrm{~m}^{3}\right)$. The proposed method produced the smallest average crown volume $\left(6.90 \mathrm{~m}^{3}\right)$.

Table 2. Crown volumes obtained using the six calculation methods *.

\begin{tabular}{|c|c|c|c|c|c|c|c|c|c|}
\hline \multirow{2}{*}{$\begin{array}{c}\text { Tree } \\
\text { Number }\end{array}$} & \multirow{2}{*}{ Tree Species } & \multirow{2}{*}{$\mathrm{CD}(\mathrm{m})$} & \multirow{2}{*}{$\mathrm{CH}(\mathrm{m})$} & \multicolumn{6}{|c|}{ Crown Volume $\left(\mathrm{m}^{3}\right)$} \\
\hline & & & & M1 & M2 & M3 & M4 & M5 & M6 \\
\hline 1 & G. biloba & 2.13 & 3.23 & 3.84 & 5.29 & 0.81 & 2.92 & 2.98 & 1.12 \\
\hline 2 & G. biloba & 4.16 & 6.18 & 28.05 & 35.65 & 6.26 & 19.93 & 13.82 & 9.59 \\
\hline 3 & G. biloba & 3.87 & 5.60 & 21.95 & 32.31 & 5.71 & 18.92 & 12.41 & 7.75 \\
\hline 4 & G. biloba & 3.82 & 5.61 & 21.48 & 32.85 & 6.04 & 19.25 & 14.96 & 11.30 \\
\hline 5 & G. biloba & 3.43 & 6.41 & 19.77 & 30.92 & 4.29 & 16.59 & 12.48 & 6.82 \\
\hline 6 & G. biloba & 3.02 & 5.32 & 12.69 & 16.41 & 1.35 & 9.17 & 5.55 & 3.83 \\
\hline 7 & G. biloba & 4.21 & 6.48 & 30.11 & 43.07 & 7.76 & 24.51 & 14.68 & 13.19 \\
\hline 8 & G. biloba & 3.61 & 6.29 & 21.46 & 33.63 & 13.67 & 21.86 & 13.66 & 12.46 \\
\hline 9 & G. biloba & 4.10 & 5.46 & 24.06 & 35.47 & 3.85 & 18.01 & 9.63 & 10.33 \\
\hline 10 & G. biloba & 3.82 & 7.12 & 27.16 & 36.69 & 6.33 & 21.09 & 11.98 & 10.55 \\
\hline 11 & P. orientalis & 2.11 & 2.85 & 6.61 & 5.61 & 1.42 & 4.96 & 2.78 & 1.81 \\
\hline 12 & P. orientalis & 3.49 & 4.27 & 27.18 & 17.89 & 8.73 & 12.36 & 6.91 & 6.38 \\
\hline 13 & P. orientalis & 2.38 & 3.64 & 10.83 & 7.43 & 2.49 & 4.13 & 3.38 & 2.32 \\
\hline 14 & P. orientalis & 3.20 & 4.17 & 22.35 & 14.83 & 7.73 & 10.82 & 5.90 & 4.22 \\
\hline 15 & P. orientalis & 4.34 & 4.17 & 41.19 & 33.21 & 19.96 & 25.18 & 6.78 & 12.69 \\
\hline 16 & P. orientalis & 3.94 & 5.59 & 45.46 & 26.58 & 12.24 & 17.95 & 8.65 & 8.54 \\
\hline 17 & P. orientalis & 2.40 & 2.99 & 9.05 & 5.97 & 1.94 & 2.99 & 1.82 & 1.45 \\
\hline 18 & P. orientalis & 3.40 & 3.67 & 22.16 & 14.75 & 8.70 & 9.80 & 4.80 & 4.20 \\
\hline 19 & P. orientalis & 3.37 & 4.39 & 26.15 & 13.16 & 5.68 & 8.98 & 5.42 & 3.46 \\
\hline 20 & P. orientalis & 2.60 & 3.60 & 12.74 & 8.90 & 2.95 & 6.99 & 3.87 & 3.03 \\
\hline 21 & P. orientalis & 1.89 & 3.34 & 6.22 & 4.89 & 1.57 & 2.17 & 1.56 & 1.03 \\
\hline 22 & P. orientalis & 2.84 & 5.85 & 24.71 & 23.28 & 14.02 & 11.76 & 4.58 & 6.30 \\
\hline 23 & P. orientalis & 2.23 & 3.78 & 9.87 & 7.27 & 3.08 & 4.55 & 3.21 & 1.97 \\
\hline 24 & P. orientalis & 2.31 & 3.82 & 10.66 & 6.65 & 1.53 & 2.83 & 2.74 & 1.59 \\
\hline 25 & P. orientalis & 3.22 & 4.55 & 24.69 & 15.86 & 4.58 & 9.07 & 5.21 & 4.67 \\
\hline 26 & P. orientalis & 4.08 & 5.42 & 47.29 & 31.91 & 19.82 & 22.52 & 8.67 & 11.07 \\
\hline 27 & P. orientalis & 1.94 & 3.45 & 6.79 & 4.30 & 1.12 & 2.40 & 2.33 & 1.50 \\
\hline 28 & P. orientalis & 3.80 & 5.42 & 41.03 & 33.23 & 17.60 & 21.71 & 9.76 & 11.93 \\
\hline 29 & P. orientalis & 3.59 & 4.48 & 30.29 & 23.22 & 8.31 & 15.42 & 6.82 & 7.87 \\
\hline 30 & P. orientalis & 4.96 & 6.64 & 85.54 & 66.58 & 50.28 & 38.90 & 10.22 & 23.99 \\
\hline \multicolumn{4}{|c|}{$\operatorname{Max}\left(\mathrm{m}^{3}\right)$} & 85.54 & 66.58 & 50.28 & 38.90 & 14.96 & 23.99 \\
\hline \multicolumn{4}{|c|}{$\operatorname{Min}\left(m^{3}\right)$} & 3.84 & 4.30 & 0.81 & 2.17 & 1.56 & 1.03 \\
\hline \multicolumn{4}{|c|}{ Mean $\left(\mathrm{m}^{3}\right)$} & 24.05 & 22.26 & 8.33 & 13.59 & 7.25 & 6.90 \\
\hline \multicolumn{4}{|c|}{ Stand. Dev. $\left(\mathrm{m}^{3}\right)$} & 16.21 & 14.54 & 9.48 & 8.74 & 4.14 & 5.09 \\
\hline
\end{tabular}

* Definitions: CD, Crown diameter; CH, Crown height; M1, Manual measurement; M2, 3D convex hull; M3, 3D alpha shape; M4, Convex hull by slices; M5, Voxel-based; M6, Concave hull by slices.

A scatter plot of crown volumes calculated by the proposed method against corresponding manual measurement values is shown in Figure 8. The crown volumes calculated using the latter method were larger than those calculated using the proposed method by an average of $17.15 \mathrm{~m}^{3}$. There was an outlier point in the scatter plot in Figure 8a, which needed to be removed to obtain robust fitting results. The scatter plot and linear fitting of crown volumes calculated by the two methods after denosing are shown in Figure $8 \mathrm{~b}$. A $t$-test on the results showed a significant difference at the $5 \%$ significance level ( $p$-value $<0.0001$ ), because the manual measurement method uses geometric entities to simulate the crown, resulting in an over-calculation of the volume [11]. By constructing a better geometric representation of the crown structure, the concave hull by slices method improved the accuracy of crown volume calculation.

A scatter plot of crown volumes calculated by the proposed method against corresponding 3D convex hull values is shown in Figure 9. As with manual measurement, the crown volumes calculated using the 3D convex hull method were larger than those calculated using the proposed method. A $t$-test on the result showed a significant difference at the $5 \%$ significance level ( $p$-value $<0.0001$ ), because the former approach calculated the volume by constructing a 3D convex geometry of the outer surface of the point cloud, which over-calculates the volume of the actual crown that included gaps and holes [9]. 

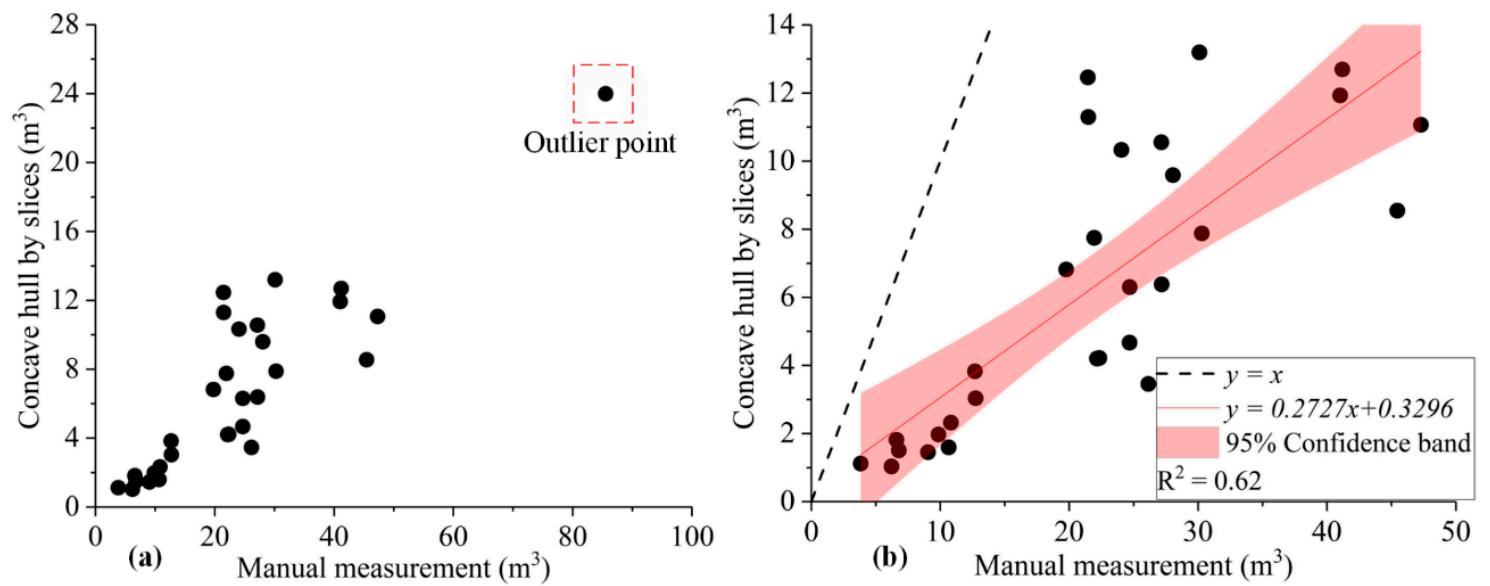

Figure 8. Scatter plot of crown volumes calculated by the concave hull by slices and manual measurement methods, where the point in the red dotted box is an outliner point (a). Scatter plot and linear fitting of crown volumes with deleted outliers are shown in (b).
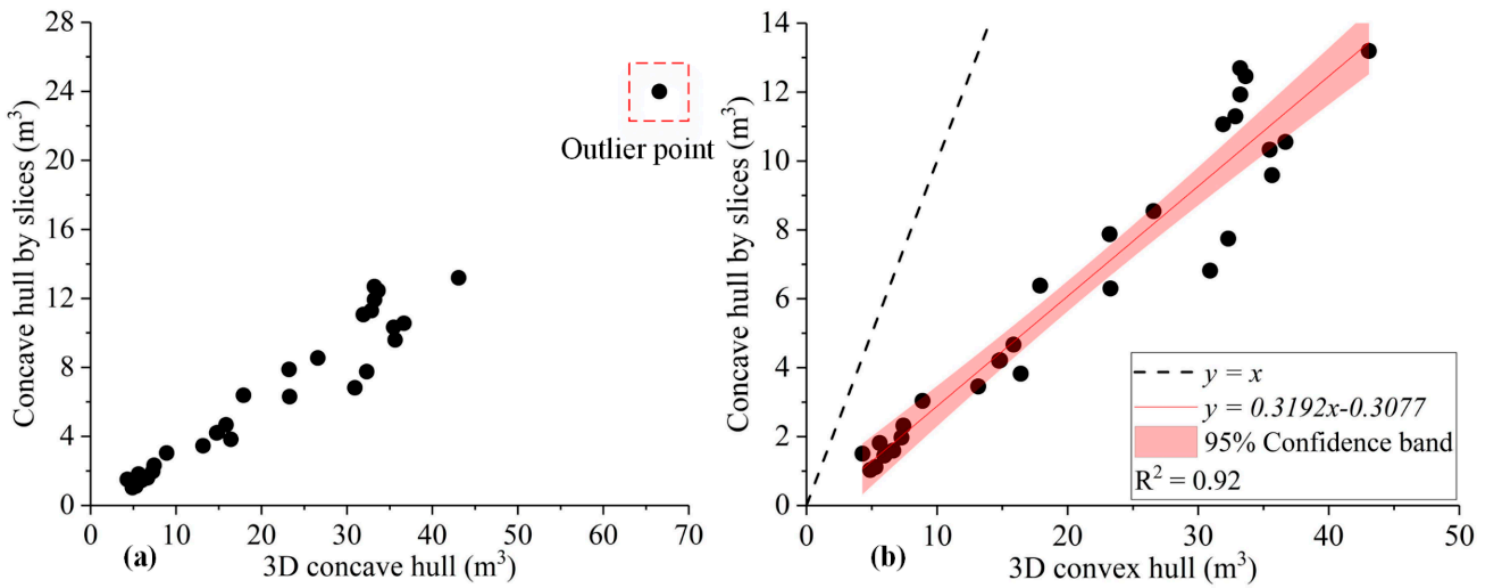

Figure 9. Scatter plot of crown volumes calculated by the concave hull by slices and 3D convex hull methods, where the point in the red dotted box is an outlier point (a). Scatter plot and linear fitting of crown volumes with deleted outliers are shown in (b).

Figure 10a shows a scatter plot of crown volumes calculated by the proposed method against corresponding 3D alpha shape values, where the point in the red dotted line box is an outlier. A $t$-test on the results of deleting the outliers showed a $p$-value of 0.6595 , greater than the critical value at the $5 \%$ significance level, implying no meaningful comparison as shown by the point distribution in Figure 10b. Further analysis reveals that the crown volumes calculated by the concave hull by slices method exceed those calculated using the 3D alpha shape approach in $90 \%$ of the G. biloba trees. There is less disparity between the $20 \mathrm{P}$. orientalis results, with the proposed method exceeding the 3D alpha shape results only in $25 \%$ of the trees and producing smaller crown volumes for 15 samples. Overall, for samples with a small crown, the crown volumes calculated using the concave hull by slices method are larger than those calculated using 3D alpha shape. VLS imaging misses more points in small-sized crowns, resulting in point clouds with more holes. The 3D alpha shape approach excessively removes holes and gaps in calculating the crown volume, resulting in under-calculation of the volumes of small crowns [42,43]; as it avoids this effect, the concave hull by slices method is more applicable across the crown size spectrum than the $3 \mathrm{D}$ alpha shape approach. 

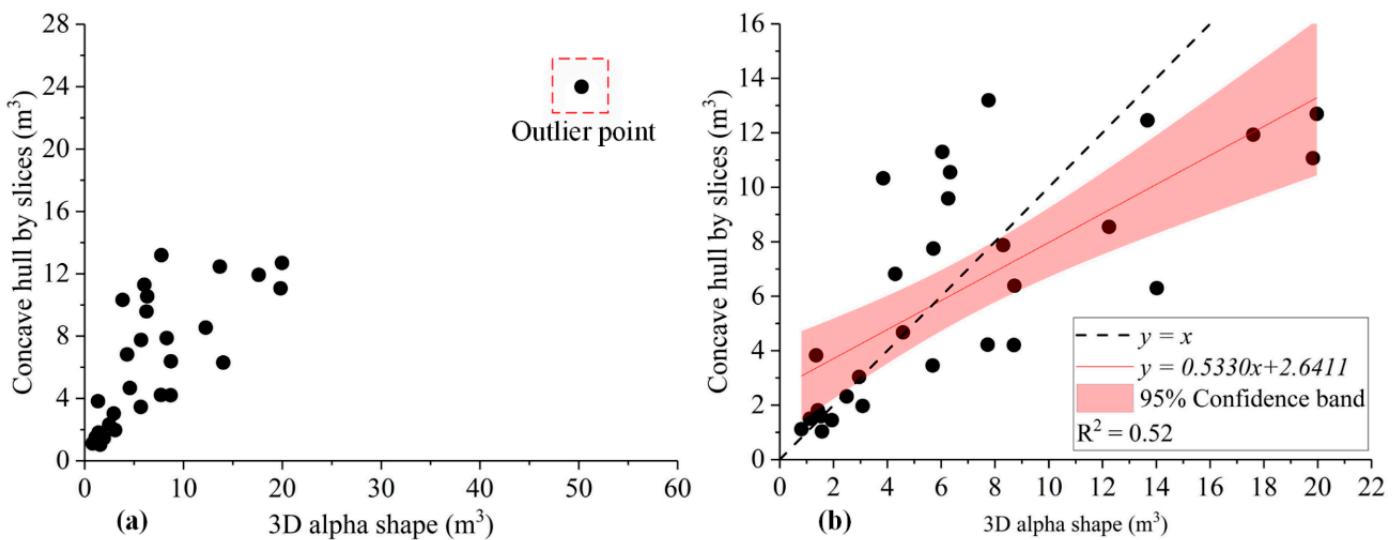

Figure 10. Scatter plot of crown volumes calculated by the concave hull by slices and 3D alpha shape methods, where the point in the red dotted box is an outlier point (a). Scatter plot and linear fitting of crown volumes with deleted outliers are shown in (b).

A scatter plot of crown volumes calculated by the proposed method against corresponding convex hull by slices values is shown in Figure 11. For a given slice point cloud, the slice plane area calculated using the convex hull approach exceeded its actual value [48]. Because the concave hull approach obtains an approximately accurate outer contour of the point cloud by constructing a concave hull (Figure 6d), it produced crown point cloud slices with volumes closer to the actual values [56,57] and less than the corresponding the convex hull results. A $t$-test on the results at the $5 \%$ significance level ( $p$-value $<0.001)$ proved that the convex hull by slices method produced larger volumes than the proposed method. The crown volumes of 30 sample trees calculated by convex hull by slices method and concave hull by slices method are smaller than that of manual measurement method and 3D convex hull method (Table 2). The latter two methods regard the crown as a whole to calculate the volume, and the volume includes all internal holes in the crown and external spaces between branches, thereby generating an overestimation of the crown volume [9]. Compared with the overall fitting strategy of the tree crown, the slice method can weaken the influence of the holes in the tree crown point cloud on the calculation results.
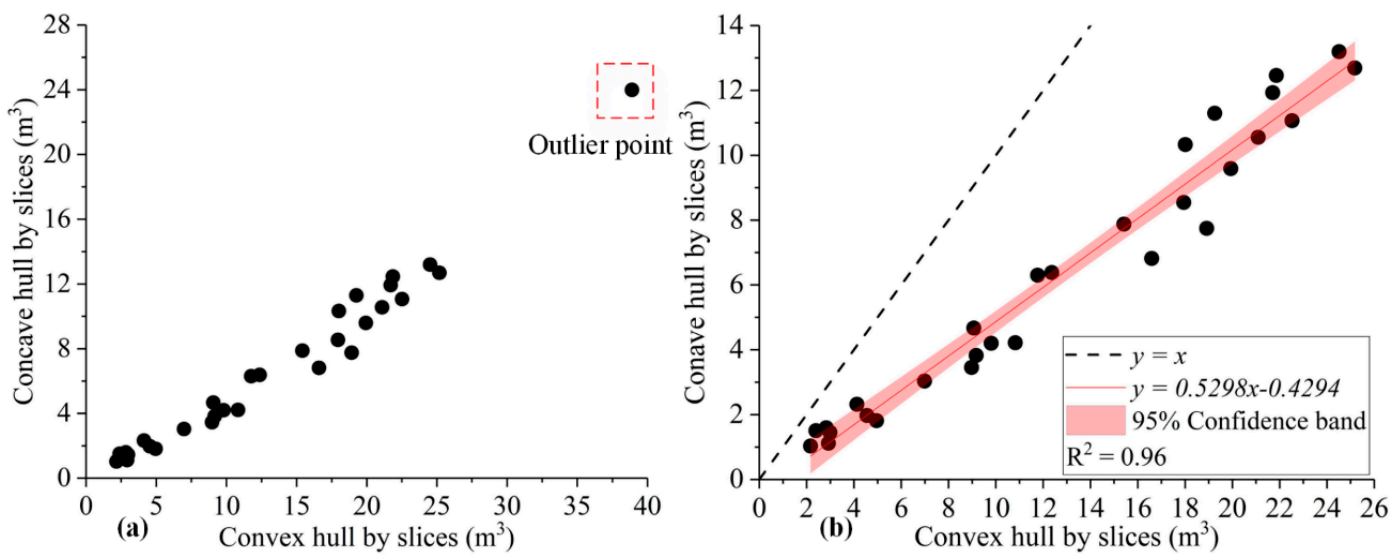

Figure 11. Scatter plot of crown volumes calculated by the concave hull by slices and convex hull by slices methods, where the point in the red dotted box is an outlier point (a). Scatter plot and linear fitting of crown volumes with deleted outliers are shown in (b).

A scatter plot of crown volumes calculated by the proposed method against the corresponding voxel-based values is shown in Figure 12. The voxel-based method can eliminate gaps and holes within the crown to some extent and so produces a useful approximation of the crown's shape. The average crown volumes calculated using this approach $\left(7.25 \mathrm{~m}^{3}\right)$ and the concave hull by slices method 
$\left(6.90 \mathrm{~m}^{3}\right)$ were the smallest among the six methods, as was the difference between the two $\left(0.35 \mathrm{~m}^{3}\right)$ (Table 2). Lecigne et al. [25] pointed out that the crown volume calculated by the voxel-based method is greatly affected by the voxel size; however, the impact of the initial slice thickness on concave hull by slices method remains to be studied. To further compare the two methods, we carried out the following experiments. First, the voxel size of the voxel-based method and the initial slice thickness of concave hull by slices method, in turn, were set from $5 \mathrm{~cm}$ to $50 \mathrm{~cm}$ with an interval of $5 \mathrm{~cm}$. Second, the two methods were applied to calculate the volume of the same tree crown point cloud, and the result is shown in Figure 13. Tree crown volume calculated by voxel-based method increased from $0.51 \mathrm{~m}^{3}$ to $32.50 \mathrm{~m}^{3}$, and the calculation results of the crown volume depend heavily on the voxel size. Finding the optimum voxel size is not easy, and the voxel-based method is likely more sensitive to the point cloud completeness. However, the crown volume calculated by concave hull by slices method fluctuated between $10 \mathrm{~m}^{3}$ and $15 \mathrm{~m}^{3}$, and was relatively less affected by the initial slice thickness. Compared with the voxel-based method, the crown volume calculated by the proposed method was more stable.
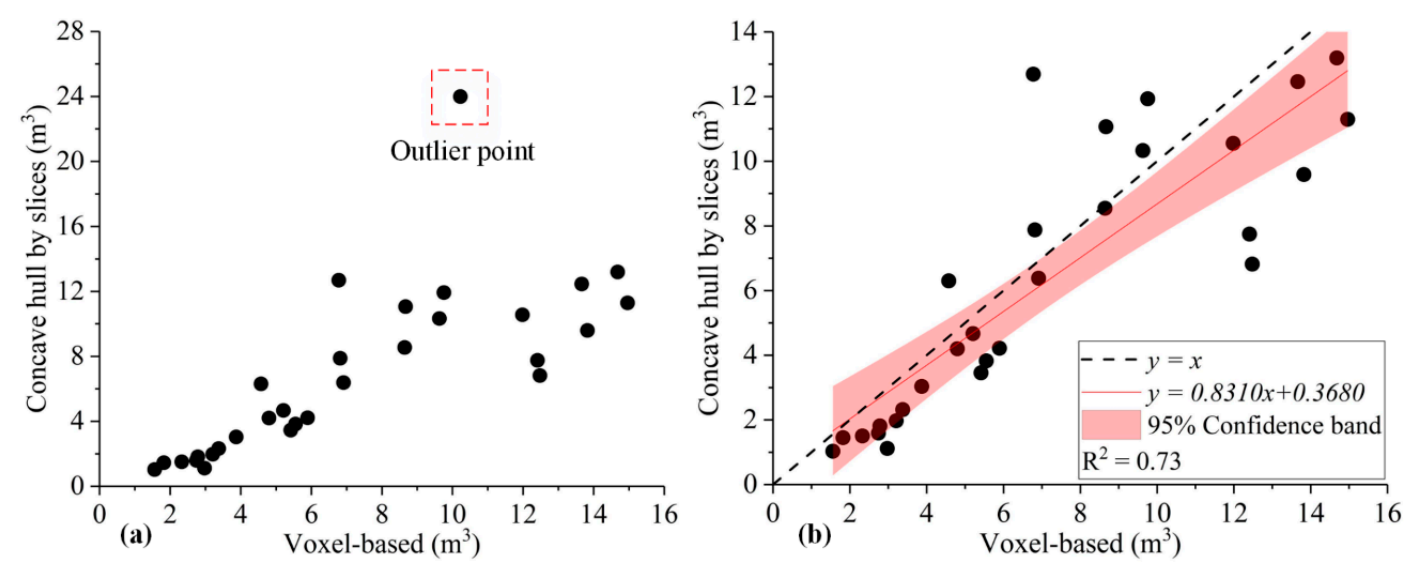

Figure 12. Scatter plot of crown volumes calculated by the concave hull by slices and voxel-based methods, where the point in the red dotted box is an outlier point (a). Scatter plot and linear fitting of crown volumes with deleted outliers are shown in (b).

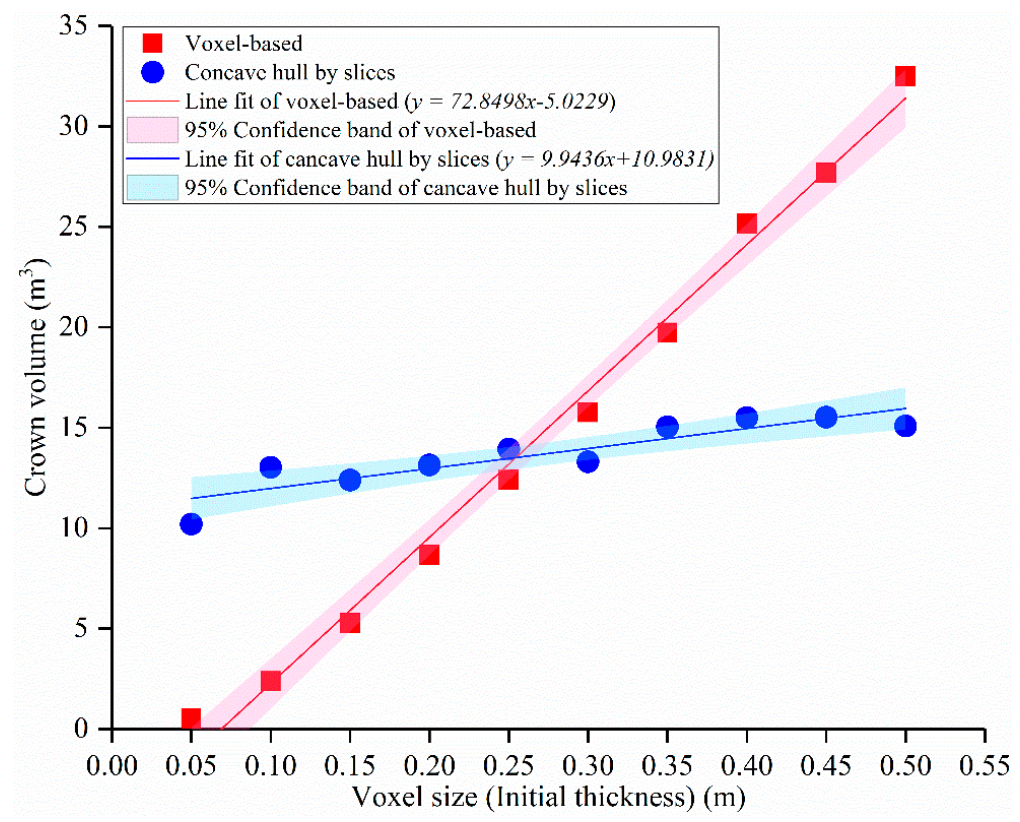

Figure 13. Comparative test of voxel-based method and concave hull by slices method. 
This study focused on calculating the crown volume of individual trees using VLS LiDAR data from common broadleaf urban trees, while excluding other important coniferous trees. In future research, we intend to further test the effectiveness of the proposed method on coniferous trees, as well as on the estimation of biomass and carbon stocks using calculated tree crown volumes. In addition, the selection of initial thickness has a certain impact on the crown volume calculated by the proposed method, and the determination of the optimal initial thickness will be carried out in future research.

\section{Conclusions}

The use of vehicle-borne laser scanning (VLS) technology is a new approach in the field of remote sensing $[29,30]$. In order to apply VLS data to the calculation of individual tree crown volumes, we developed a concave hull by slices method that improves on existing methods. To validate this approach, the crown volumes of 30 single urban trees ( $10 \mathrm{G}$. biloba and 20 P. orientalis) were calculated using the proposed method and compared with the results produced by five existing methods. These results indicate that the proposed method is best suited among the examined ones to calculate the volume of individual tree crowns with VLS data, to be used in the subsequent estimation of biomass and carbon stocks. This study can be summarized as follows:

(1) Since gaps and holes exist in the crown, the calculated volume will be larger than the true value without removal of these spaces. The pre-existing convex hull method cannot eliminate this effect, resulting in an overly large calculated crown volume, while the concave hull by slices method can address this problem to a certain extent, improving the accuracy of crown volume estimation.

(2) An adaptive slicing method based on the rate of change of crown area with height was proposed to reduce the number of slice layers and ensure that the thickness of each slice and the number of slice layers were adapted to the shape and size of the tree crown itself, improving the slicing rationality of the point cloud in the vertical direction.

(3) The proposed concave hull by slice method produced the smallest average estimated crown volume $\left(6.90 \mathrm{~m}^{3}\right)$ of the six tested methods. Manual measurement, the 3D convex hull method, and the convex hull by slices method produced volumes that were consistently higher than the proposed method. The 3D alpha shape method and voxel-based method showed a fair agreement on the average values and the proposed method and voxel-based method showed more internal coherence, as they have low standard deviation and low ranges compare to the others. However, the proposed method is likely more robust than voxel-based method.

Author Contributions: Conceptualization, Z.Y., R.L., and L.C.; Data curation, Z.Y., X.Z., X.R., and Y.X.; Formal analysis, Z.Y., X.Z., X.R., and Y.X.; Funding acquisition, Z.Y. and L.C.; Investigation, Z.Y., X.Z., and X.R.; Methodology, Z.Y., R.L., and L.C.; Project administration, Z.Y., R.L., and L.C.; Resources, L.C.; Software, Z.Y., X.Z., X.R., and Y.X.; Supervision, R.L. and L.C.; Validation, R.L. and L.C.; Visualization, Z.Y., X.Z., and X.R.; Writing original draft, Z.Y., R.L., L.C., X.Z., X.R., and Y.X.; Writing review \& editing, Z.Y., R.L., L.C., X.Z., X.R., and Y.X.

Funding: This research was funded by the National Key R\&D Program of China, grant number [2017YFB0504205], and the National Natural Science Foundation of China, grant number [41622109, 41371017].

Acknowledgments: We wish to express our great appreciation to Qingdao Xiushan Mobile Measurement Co., Ltd. for providing research data.

Conflicts of Interest: The authors declare no conflict of interest.

\section{References}

1. Bao, Y.; Tian, Q.; Chen, M.; Lin, H. An automatic extraction method for individual tree crowns based on self-adaptive mutual information and tile computing. Int. J. Digit. Earth 2014, 8, 495-516. [CrossRef]

2. Newbold, T.; Hudson, L.N.; Hill, S.L.; Contu, S.; Lysenko, I.; Senior, R.A.; Borger, L.; Bennett, D.J.; Choimes, A.; Collen, B.; et al. Global effects of land use on local terrestrial biodiversity. Nature 2015, 520, 45-50. [CrossRef]

3. Fang, F.; Im, J.; Lee, J.; Kim, K. An improved tree crown delineation method based on live crown ratios from airborne LiDAR data. GISci. Remote Sens. 2016, 53, 402-419. [CrossRef] 
4. Alexander, C.; Korstjens, A.H.; Hill, R.A. Influence of micro-topography and crown characteristics on tree height estimations in tropical forests based on LiDAR canopy height models. Int. J. Appl. Earth Obs. Geoinf. 2018, 65, 105-113. [CrossRef]

5. Dalponte, M.; Frizzera, L.; Ørka, H.O.; Gobakken, T.; Næsset, E.; Gianelle, D. Predicting stem diameters and aboveground biomass of individual trees using remote sensing data. Ecol. Indic. 2018, 85, 367-376. [CrossRef]

6. Wu, B.; Yu, B.; Wu, Q.; Huang, Y.; Chen, Z.; Wu, J. Individual tree crown delineation using localized contour tree method and airborne LiDAR data in coniferous forests. Int. J. Appl. Earth Obs. Geoinf. 2016, 52, 82-94. [CrossRef]

7. Xu, W.H.; Feng, Z.K.; Su, Z.F.; Xu, H.; Jiao, Y.Q.; Deng, O. An automatic extraction algorithm for individual tree crown projection area and volume based on 3D point cloud data. Spectrosc. Spectr. Anal. 2014, 34, 465-471.

8. Phattaralerphong, J.; Sinoquet, H. A method for 3D reconstruction of tree crown volume from photographs: Assessment with 3D-digitized plants. Tree Physiol. 2005, 25, 1229-1242. [CrossRef]

9. Fernández-Sarría, A.; Martínez, L.; Velázquez-Martí, B.; Sajdak, M.; Estornell, J.; Recio, J.A. Different methodologies for calculating crown volumes of Platanus hispanica trees using terrestrial laser scanner and a comparison with classical dendrometric measurements. Comput. Electron. Agric. 2013, 90, 176-185. [CrossRef]

10. Korhonen, L.; Vauhkonen, J.; Virolainen, A.; Hovi, A.; Korpela, I. Estimation of tree crown volume from airborne lidar data using computational geometry. Int. J. Remote Sens. 2013, 34, 7236-7248. [CrossRef]

11. Lin, W.; Meng, Y.; Qiu, Z.; Zhang, S.; Wu, J. Measurement and calculation of crown projection area and crown volume of individual trees based on 3D laser-scanned point-cloud data. Int. J. Remote Sens. 2017, 38, 1083-1100. [CrossRef]

12. Lin, Y.; Jaakkola, A.; Hyyppä, J.; Kaartinen, H. From TLS to VLS: Biomass Estimation at Individual Tree Level. Remote Sens. 2010, 2, 1864-1879. [CrossRef]

13. Rautiainen, M.; Mõttus, M.; Stenberg, P.; Ervasti, S. Crown Envelope Shape Measurements and Models. Silva Fenn. 2008, 42, 19-33. [CrossRef]

14. Paris, C.; Kelbe, D.; Aardt, J.V.; Bruzzone, L. A Novel Automatic Method for the Fusion of ALS and TLS LiDAR Data for Robust Assessment of Tree Crown Structure. IEEE Trans. Geosci. Remote Sens. 2017, 55, 1-15. [CrossRef]

15. Jing, L.; Hu, B.; Noland, T.; Li, J. An individual tree crown delineation method based on multi-scale segmentation of imagery. ISPRS J. Photogramm. Remote Sens. 2012, 70, 88-98. [CrossRef]

16. Yin, W.; Yang, J.; Yamamoto, H.; Li, C. Object-based larch tree-crown delineation using high-resolution satellite imagery. Int. J. Remote Sens. 2015, 36, 822-844. [CrossRef]

17. Varekamp, C.; Hoekman, D.H. High-resolution InSAR image simulation for forest canopies. IEEE Trans. Geosci. Remote Sens. 2002, 40, 1648-1655. [CrossRef]

18. Dalponte, M.; Ene, L.T.; Marconcini, M.; Gobakken, T.; Næsset, E. Semi-supervised SVM for individual tree crown species classification. ISPRS J. Photogramm. Remote Sens. 2015, 110, 77-87. [CrossRef]

19. Liu, T.; Im, J.; Quackenbush, L.J. A novel transferable individual tree crown delineation model based on Fishing Net Dragging and boundary classification. ISPRS J. Photogramm. Remote Sens. 2015, 110, $34-47$. [CrossRef]

20. Tochon, G.; Féret, J.B.; Valero, S.; Martin, R.E.; Knapp, D.E.; Salembier, P.; Chanussot, J.; Asner, G.P. On the use of binary partition trees for the tree crown segmentation of tropical rainforest hyperspectral images. Remote Sens. Environ. 2015, 159, 318-331. [CrossRef]

21. Strîmbu, V.F.; Strîmbu, B.M. A graph-based segmentation algorithm for tree crown extraction using airborne LiDAR data. ISPRS J. Photogramm. Remote Sens. 2015, 104, 30-43. [CrossRef]

22. Leckie, D.G.; Walsworth, N.; Gougeon, F.A. Identifying tree crown delineation shapes and need for remediation on high resolution imagery using an evidence based approach. ISPRS J. Photogramm. Remote Sens. 2016, 114, 206-227. [CrossRef]

23. Kukunda, C.B.; Duque-Lazo, J.; González-Ferreiro, E.; Thaden, H.; Kleinn, C. Ensemble classification of individual Pinus crowns from multispectral satellite imagery and airborne LiDAR. Int. J. Appl. Earth Obs. Geoinf. 2018, 65, 12-23. [CrossRef] 
24. Kato, A.; Moskal, L.M.; Schiess, P.; Swanson, M.E.; Calhoun, D.; Stuetzle, W. Capturing tree crown formation through implicit surface reconstruction using airborne lidar data. Remote Sens. Environ. 2009, 113, 1148-1162. [CrossRef]

25. Lecigne, B.; Delagrange, S.; Messier, C. Exploring trees in three dimensions: VoxR, a novel voxel-based R package dedicated to analysing the complex arrangement of tree crowns. Ann. Bot. 2018, 121, 589-601. [CrossRef]

26. Miranda-Fuentes, A.; Llorens, J.; Gamarra-Diezma, J.L.; Gil-Ribes, J.A.; Gil, E. Towards an optimized method of olive tree crown volume measurement. Sensors 2015, 15, 3671-3687. [CrossRef]

27. Gong, Y.X.; Fei, Y.; Feng, Z.K.; Liu, Y.F.; Xue, W.X.; Fei, X.; University, B.F. Extraction of crown volume using triangulated irregular network algorithm based on LiDAR. J. Infrared Millim. Waves 2016, 35, 177-183.

28. Cabo, C.; Ordoñez, C.; García-Cortés, S.; Martínez, J. An algorithm for automatic detection of pole-like street furniture objects from Mobile Laser Scanner point clouds. ISPRS J. Photogramm. Remote Sens. 2014, 87, 47-56. [CrossRef]

29. Yang, B.; Dong, Z.; Liu, Y.; Liang, F.; Wang, Y. Computing multiple aggregation levels and contextual features for road facilities recognition using mobile laser scanning data. ISPRS J. Photogramm. Remote Sens. 2017, 126, 180-194. [CrossRef]

30. Wang, J.; Lindenbergh, R.; Menenti, M. SigVox-A 3D feature matching algorithm for automatic street object recognition in mobile laser scanning point clouds. ISPRS J. Photogramm. Remote Sens. 2017, 128, 111-129. [CrossRef]

31. Li, L.; Li, D.; Zhu, H.; Li, Y. A dual growing method for the automatic extraction of individual trees from mobile laser scanning data. ISPRS J. Photogramm. Remote Sens. 2016, 120, 37-52. [CrossRef]

32. Wang, Y.; Cheng, L.; Chen, Y.; Wu, Y.; Li, M. Building Point Detection from Vehicle-Borne LiDAR Data Based on Voxel Group and Horizontal Hollow Analysis. Remote Sens. 2016, 8, 419. [CrossRef]

33. Kukko, A.; Kaartinen, H.; Hyyppä, J.; Chen, Y. Multiplatform Mobile Laser Scanning: Usability and Performance. Sensors 2012, 12, 11712-11733. [CrossRef]

34. Haala, N.; Peter, M.; Kremer, J.; Hunter, G. Mobile LiDAR mapping for 3D point cloud collection in urban areas-A performance test. Int. Arch. Photogramm. Remote Sens. Spat. Inf. Sci 2008, 37, 1119-1127.

35. Wu, B.; Yu, B.; Yue, W.; Shu, S.; Tan, W.; Hu, C.; Huang, Y.; Wu, J.; Liu, H. A Voxel-Based Method for Automated Identification and Morphological Parameters Estimation of Individual Street Trees from Mobile Laser Scanning Data. Remote Sens. 2013, 5, 584-611. [CrossRef]

36. Schumann, A.W. Performance of an Ultrasonic Tree Volume Measurement System in Commercial Citrus Groves. Precis. Agric. 2005, 6, 467-480.

37. Gupta, S.; Weinacker, H.; Koch, B. Comparative Analysis of Clustering-Based Approaches for 3-D Single Tree Detection Using Airborne Fullwave Lidar Data. Remote Sens. 2010, 2, 968-989. [CrossRef]

38. Olsoy, P.J.; Glenn, N.F.; Clark, P.E.; Derryberry, D.R. Aboveground total and green biomass of dryland shrub derived from terrestrial laser scanning. ISPRS J. Photogramm. Remote Sens. 2014, 88, 166-173. [CrossRef]

39. Vauhkonen, J.; Tokola, T.; Packalén, P.; Maltamo, M. Identification of Scandinavian commercial species of individual trees from airborne laser scanning data using alpha shape metrics. For. Sci. 2009, 55, 37-47.

40. Vauhkonen, J.; Korpela, I.; Maltamo, M.; Tokola, T. Imputation of single-tree attributes using airborne laser scanning-based height, intensity, and alpha shape metrics. Remote Sens. Environ. 2010, 114, 1263-1276. [CrossRef]

41. Yao, W.; Krzystek, P.; Heurich, M. Tree species classification and estimation of stem volume and DBH based on single tree extraction by exploiting airborne full-waveform LiDAR data. Remote Sens. Environ. 2012, 123, 368-380. [CrossRef]

42. Vauhkonen, J.; Seppänen, A.; Packalén, P.; Tokola, T. Improving species-specific plot volume estimates based on airborne laser scanning and image data using alpha shape metrics and balanced field data. Remote Sens. Environ. 2012, 124, 534-541. [CrossRef]

43. Zhen, Z.; Quackenbush, L.; Zhang, L. Trends in Automatic Individual Tree Crown Detection and Delineation-Evolution of LiDAR Data. Remote Sens. 2016, 8, 333. [CrossRef]

44. Vauhkonen, J.; Tokola, T.; Maltamo, M.; Packalén, P. Effects of pulse density on predicting characteristics of individual trees of Scandinavian commercial species using alpha shape metrics based on airborne laser scanning data. Can. J. Remote Sens. 2014, 34, S441-S459. [CrossRef] 
45. Popescu, S.C.; Zhao, K. A voxel-based lidar method for estimating crown base height for deciduous and pine trees. Remote Sens. Environ. 2008, 112, 767-781. [CrossRef]

46. Hosoi, F.; Omasa, K. Voxel-Based 3-D Modeling of Individual Trees for Estimating Leaf Area Density Using High-Resolution Portable Scanning Lidar. IEEE Trans. Geosci. Remote Sens. 2006, 44, 3610-3618. [CrossRef]

47. Hyyppa, J.; Kelle, O.; Lehikoinen, M.; Inkinen, M. A segmentation-based method to retrieve stem volume estimates from 3-D tree height models produced by laser scanners. IEEE Trans. Geosci. Remote Sens. 2001, 39, 969-975. [CrossRef]

48. Moreira, A.J.C.; Santos, M.Y. Concave hull: A k-nearest neighbours approach for the computation of the region occupied by a set of points. In Proceedings of the Second International Conference on Computer Graphics Theory and Applications (GRAPP 2007), Barcelona, Spain, 8-11 March 2007; Volume GM/R, pp. 61-68.

49. Zhong, L.; Cheng, L.; Xu, H.; Wu, Y.; Chen, Y.; Li, M. Segmentation of Individual Trees from TLS and MLS Data. IEEE J. Sel. Top. Appl. Earth Observ. Remote Sens. 2017, 10, 774-787. [CrossRef]

50. Pourkhabbaz, A.; Rastin, N.; Olbrich, A.; Langenfeld-Heyser, R.; Polle, A. Influence of environmental pollution on leaf properties of urban plane trees, Platanus orientalis L. Bull. Environ. Contam. Toxicol. 2010, 85, 251-255. [CrossRef]

51. Guan, R.; Zhao, Y.; Zhang, H.; Fan, G.; Liu, X.; Zhou, W.; Shi, C.; Wang, J.; Liu, W.; Liang, X.; et al. Draft genome of the living fossil Ginkgo biloba. GigaScience 2016, 5, 49. [CrossRef]

52. Yin, S.; Shen, Z.; Zhou, P.; Zou, X.; Che, S.; Wang, W. Quantifying air pollution attenuation within urban parks: An experimental approach in Shanghai, China. Environ. Pollut. 2011, 159, 2155-2163. [CrossRef]

53. CloudCompare. CloudCompare (Version 2.8). 2017. Available online: http://www.cloudcompare.org/ (accessed on 12 May 2018).

54. Dewez, T.J.B.; Girardeau-Montaut, D.; Allanic, C.; Rohmer, J. Facets: A Cloudcompare Plugin to Extract Geological Planes from Unstructured 3d Point Clouds. ISPRS Arch. 2016, XLI-B5, 799-804.

55. Stovall, A.E.L.; Vorster, A.G.; Anderson, R.S.; Evangelista, P.H.; Shugart, H.H. Non-destructive aboveground biomass estimation of coniferous trees using terrestrial LiDAR. Remote Sens. Environ. 2017, 200, 31-42. [CrossRef]

56. Park, J.S.; Oh, S.J. A New Concave Hull Algorithm and Concaveness Measure for n-dimensional Datasets. J. Inf. Sci. Eng. 2013, 29, 587-600.

57. Asaeedi, S.; Didehvar, F.; Mohades, A. $\alpha$-Concave hull, a generalization of convex hull. Theor. Comput. Sci. 2017, 702, 48-59. [CrossRef]

58. Cheng, L.; Wu, Y.; Chen, S.; Zong, W.; Yuan, Y.; Sun, Y.; Zhuang, Q.; Li, M. A symmetry-based method for Lidar point registration. IEEE J. Sel. Top. Appl. Earth Observ. Remote Sens. 2018, 11, 285-299. [CrossRef]

59. Hosoi, F.; Nakai, Y.; Omasa, K. 3-D voxel-based solid modeling of a broad-leaved tree for accurate volume estimation using portable scanning lidar. ISPRS J. Photogramm. Remote Sens. 2013, 82, 41-48. [CrossRef] 